International Journal of Cooperative Information Systems

Vol. 21, No. 4 (2012) 405-406

(C) World Scientific Publishing Company

DOI: $10.1142 / \mathrm{S} 0218843012990018$

\title{
AUTHOR INDEX \\ VOLUME 21 (2012)
}

Ahn, G.-J., see Zhu, Y.

Andrikopoulos, V., see Mokhtari-Aslaoui, K.

21 (2012) 165-197

21 (2012) 85-109

Benbernou, S., see Mokhtari-Aslaoui, K.

21 (2012) 85-109

Bhawna, S., see Parimala, N.

De Giacomo, G., De Masellis, R. and Rosati, R., Verification of conjunctive artifact-centric services

21 (2012) 141-164

De Masellis, R., see De Giacomo, G.

Dustdar, S., see Skopik, F.

Elgammal, A., Turetken, O. and van den Heuvel, W.-J., Using patterns for the analysis and resolution of compliance violations

Filatova, E.,Information overlap in multilingual Wikipedia and summarization

Gericke, L., Gumienny, R. and Meinel, C., Collaboratecom special issue analyzing distributed whiteboard interactions

Gumienny, R., see Gericke, L.

Hacid, M.-S., see Mokhtari-Aslaoui, K.

Hess, J., Reuter, C., Pipek, V. and Wulf, V., Supporting end-user articulations in evolving business processes: A case study to explore intuitive notations and interaction designs

$\mathrm{Hu}, \mathrm{H}$., see Zhu, Y.

Ignat, C.-L., see Truong, H. T. T.

Janssen, M., see Overbeek, S.

Kern, R., Thies, H., Zirpins, C. and Satzger, G., Dynamic and goal-based quality management for human-based electronic services

Leymann, F., see Mokhtari-Aslaoui, K.

Ma, D., see Zhu, Y.

Meinel, C., see Gericke, L.

Mendling, J., see Smirnov, S.

Mokhtari-Aslaoui, K., Benbernou, S., Sahri, S., Andrikopoulos, V., Leymann, F. and Hacid, M.-S., Timed privacy-aware business protocols

Molli, P., see Truong, H. T. T.

Overbeek, S., Janssen, M. and Tan, Y.-H., An event-driven architecture for integrating information, processes and services in a plastic toys supply chain

Parimala, N. and Bhawna, S., Continuous multiple OLAP queries for data streams

Pipek, V., see Hess, J.

21 (2012) 111-139

21 (2012) 111-139

21 (2012) 297-341

$21(2012) 31-54$

21 (2012) 483-403

21 (2012) 199-220

21 (2012) 199-220

21 (2012) 85-109

21 (2012) 263-296

21 (2012) 165-197

21 (2012) 221-262

21 (2012) 343-381

21 (2012) 3-29

21 (2012) 85-109

21 (2012) 165-197

21 (2012) 199-220

21 (2012) 55-83

21 (2012) 85-109

21 (2012) 221-262

21 (2012) 343-381

21 (2012) 141-164

21 (2012) 263-296 
Reuter, C., see Hess, J.

21 (2012) 263-296

Rosati, R., see De Giacomo, G.

21 (2012) 111-139

Sahri, S., see Mokhtari-Aslaoui, K.

21 (2012) 85-109

Satzger, G., see Kern, R.

21 (2012) 3-29

Schall, D., see Skopik, F.

21 (2012) 297-341

Skopik, F., Schall, D. and Dustdar, S., Discovering and managing social compositions in collaborative enterprise crowdsourcing systems

21 (2012) 297-341

Smirnov, S., Weidlich, M. and Mendling, J., Business process model abstraction based on synthesis from well-structured behavioral profiles

Tan, Y.-H., see Overbeek, S.

Thies, H., see Kern, R.

Truong, H. T. T., Ignat, C.-L. and Molli, P., A contract-extended push-pull-clone model for multi-synchronous collaboration

Turetken, O., see Elgammal, A.

van den Heuvel, W.-J., see Elgammal, A.

Wang, S., see Zhu, Y.

Weidlich, M., see Smirnov, S.

Wulf, V., see Hess, J.

Zhu, Y., Wang, S., Hu, H., Ahn, G.-J. and Ma, D., Secure collaborative integrity verification for hybrid cloud environments

21 (2012) 165-197

Zirpins, C., see Kern, R.

21 (2012) 3-29

21 (2012) 55-83

21 (2012) 343-381

21 (2012) 3-29

21 (2012) 221-262

21 (2012) 31-54

21 (2012) 31-54

21 (2012) 165-197

21 (2012) 55-83

21 (2012) 263-296 